\title{
Further studies of clinical isolates of cysteine-requiring Escherichia coli and Klebsiella and possible mechanisms for their selection in vivo
}

\author{
C. J. McIVER and J. W. TAPSALL \\ Department of Microbiology, The Prince of Wales Hospital, Randwick, Sydney, NSW, Australia 2031
}

\begin{abstract}
Summary. Cysteine-dependent (cys ${ }^{-}$) Escherichia coli and Klebsiella spp., defective in sulphate assimilation, were isolated from urine and stool samples of infected patients. These isolates reverted to prototrophy under conditions of cysteine deprivation but the revertant strains and a prototrophic wild-type $E$. coli strain became auxotrophic for cysteine in a cysteine-enriched medium. This suggested that excess cysteine acts as a repressor of the cys $H I J$ operon known to control aspects of cysteine biosynthesis. A group of mostly elderly patients infected with cys ${ }^{-}$strains suffered a disproportionate amount of renal impairment as compared with a control group. In renal impairment, sulphur compounds, including cysteine, are retained. This raises the possibility that these raised levels of cysteine and related compounds may enhance the selection of cys $^{-}$strains in vivo.
\end{abstract}

\section{Introduction}

About $1.5 \%$ of Escherichia coli and Klebsiella isolates from urinary tract infections (UTI) are auxotrophic for cysteine $\left(\mathrm{cys}^{-}\right) .^{1,2}$ Such strains may also occasionally cause septicaemia and other forms of sepsis, for which the urinary tract is usually the source. ${ }^{3}$ Cys $^{-}$E. coli and Klebsiella strains have an altered pathway of sulphate assimilation..$^{2,3}$ Their requirement for cysteine, and other characteristics relating to identification and antibiotic susceptibility testing, have been investigated previously. ${ }^{4-6}$ Patients infected with these strains are usually elderly, with a systemic or local predisposition to UTI. ${ }^{2,3,7}$

Since most UTI are due to bowel bacteria, stool samples from patients with cys ${ }^{-} E$. coli UTI were examined for the presence of such organisms. An invitro method, with conditions of cysteine excess, was devised to derive a cys ${ }^{-} E$. coli variant from a prototrophic strain originally cultured from a patient whose faeces also contained a cys $^{-}$isolate. The previously observed ability of cys ${ }^{-}$strains to revert to prototrophy under conditions of cysteine deprivation ${ }^{6}$ was also further investigated. It has been noted previously that cys ${ }^{-}$strains may produce colonies of differing sizes on a single culture plate, giving the appearance of a mixed growth. ${ }^{7}$ Therefore, the sulphur assimilation pathway of these different colonial types was investigated to determine whether reversion to prototrophy is a single or a multiple step process.

Analysis of the clinical findings of patients infected with cys $^{-}$strains suggested that renal impairment was

Received 9 Nov. 1992; revised version accepted 6 May 1993. a common feature in this group. Since sulphurcontaining compounds are known to be retained in patients with renal impairment, ${ }^{8-10}$ this and other observations were used to advance a hypothesis to explain the possible origin of $\mathrm{cys}^{-}$strains in vivo.

\section{Materials and methods}

\section{Faeces samples}

Stool samples were collected from 11 patients in whose blood or urine cys ${ }^{-}$E. coli or Klebsiella strains had been detected by methods described previously., Four of these specimens were collected before antibiotic treatment had been started. Samples of faeces from 100 patients aged $\geqslant 60$ years with diarrhoeal disease, but without UTI, were also collected and examined for the presence of cys ${ }^{-}$strains.

\section{Isolation of cys $^{-}$strains}

$\mathrm{Cys}^{-}$strains were isolated from faeces samples by a direct or an antibiotic-selection method. For the direct method a small amount faeces was inoculated on to MacConkey Agar (Oxoid) which was incubated at $37^{\circ} \mathrm{C}$ for $18 \mathrm{~h}$. The plates were examined for dwarf colonies characteristic of cys $^{-}$auxotrophs. These were stained by Gram's method and their cysteine requirement was determined.

For the antibiotic-selection method, faeces were seeded into a cysteine-free, penicillin-supplemented broth in which cys ${ }^{-}$strains will not grow and should, therefore, be indifferent to the action of penicillin, while actively dividing prototrophs will be greatly 
reduced in numbers. ${ }^{11}$ Faeces $(0.5 \mathrm{~g})$ were suspended in Davis and Mingioli (DM) medium ${ }^{12}$ supplemented to $0.63 \mathrm{~mm}$ with L-cysteine $\mathrm{HCl}$. After overnight incubation, the broth was filtered through sterile gauze to remove solid material and then centrifuged at $1600 \mathrm{~g}$ for $10 \mathrm{~min}$. The deposit was washed three times with saline and a 3-ml suspension of this was used to inoculate $20 \mathrm{ml}$ of cysteine-free $\mathrm{DM}$ broth at $37^{\circ} \mathrm{C}$ to an initial absorbance of $0 \cdot 15-0.25$ at $640 \mathrm{~nm}$ (Spectronic-R 20 series spectrophotometer; Milton Roy, Rochester, NY, USA). After incubation for $2 \mathrm{~h}$ in a shaking water bath, penicillin was added to $10000 \mathrm{IU} / \mathrm{ml}$ and incubation continued for a further $2 \mathrm{~h}$. The growth was then harvested as above and 10 serial 10-fold dilutions were made in saline. Each dilution was used to flood MacConkey agar plates which were incubated for $48 \mathrm{~h}$ and examined for the presence of cys $^{-}$strains as described above.

\section{Growth response of cys ${ }^{-}$colonial variants to intermediates of the sulphate assimilatory pathway}

Colonial variants of two cys ${ }^{-}$E. coli strains (ECC-1 and 13) and one cys ${ }^{-}$Klebsiella strain (KC-6) of the class cys $A C D H$ of Jones-Mortimer ${ }^{2,3,13}$ were selected on the basis of their colony size after growth for $24 \mathrm{~h}$ on MacConkey agar. Dwarf colonies $(0.5-1.0 \mathrm{~mm}$ in diameter), intermediate colonies (partial revertants; $1.5-2.0 \mathrm{~mm}$ in diameter) and larger colonies (complete revertants; $\geqslant 2.0 \mathrm{~mm}$ in diameter) were selected and inoculated into DM broth containing $0.63 \mathrm{mM} \mathrm{L}$-cysteine $\mathrm{HCl}$. After incubation for $24 \mathrm{~h}$ at $37^{\circ} \mathrm{C}$, the growth was harvested, washed three times in saline, inoculated at an initial absorbance of 0.1 at $640 \mathrm{~nm}$ into 2.5-ml volumes of Pasternak' ${ }^{14}$ sulphur-free basal medium (PM) with or without $10 \mathrm{mM} \mathrm{Na}_{2} \mathrm{SO}_{4}, 0.5 \mathrm{mM}$ L-cysteine sulphinic acid (CSH), $0.42 \mathrm{~mm} \mathrm{Na}{ }_{2} \mathrm{~S}$ or $0.63 \mathrm{mM} \mathrm{L}$-cysteine $\mathrm{HCl}$ and incubated for $8 \mathrm{~h}$ at $37^{\circ} \mathrm{C}$ in a shaking water bath. The growth of each strain in each medium was determined by measuring absorbance at 30-40-min intervals. Prototrophic E. coli NCTC 10418 and K. pneumoniae ATCC 12657 were used as controls. These experiments were performed in duplicate and repeated once.

Mutants deficient in sulphate permease, controlled by $\operatorname{cys} A$, may be identified by their failure to assimilate relatively low levels of thiosulphate. ${ }^{15,16}$ Therefore, the above strains were included in a batch of $34 \mathrm{cys}^{-}$strains of class cys $A C D H$ (32 E. coli and two K. pneumoniae), isolated and phenotyped in this laboratory. They were examined for their capacity to grow on PM containing $0.2 \mathrm{~mm}$ thiosulphate. Only dwarf variants of cys $^{-}$phenotype were examined in this experiment, which also tested their ability to grow on sulphur-free PM and on PM containing $10 \mathrm{mM} \mathrm{Na}_{2} \mathrm{SO}_{4}$.

\section{Rate of reversion of cys ${ }^{-}$strains to complete and "partial" prototrophy}

Five cys- strains isolated in this laboratory-four $E$. coli (ECC-1, 5, 13 and 21) and K. pneumoniae KC6-were used. Dwarf colonies of these strains, grown on MacConkey agar overnight, were suspended in $2.5 \mathrm{ml}$ of sterile saline to an absorbance of $c .0 .2$ at $640 \mathrm{~nm}$. Each suspension was serially diluted 100 -fold three times and $0.2 \mathrm{ml}$ of each dilution was inoculated on to separate plates of PM supplemented with $10 \mathrm{mM} \mathrm{Na}_{2} \mathrm{SO}_{4}, 0.5 \mathrm{~mm} \mathrm{CSH}$ or $0.63 \mathrm{~mm}$ cysteine. Plates were incubated for 6 days in air at $37^{\circ} \mathrm{C}$. Total counts were made of the revertant colonies appearing on plates supplemented with sulphate and CSH and the count on the latter plate corrected for the presence of complete revertants (prototrophs) on the sulphate plate. A ratio of counts of partial (corrected CSH growth) and complete (growth in the presence of sulphate) revertants to the viable count of the initial inoculum (determined by surface viable counts on cysteine-supplemented PM) was calculated. A mean of the rates of partial and complete reversion to prototrophy was determined after repeating the experiment four times.

\section{Derivation of cys strains from prototrophic strains}

A single colony of the strain under examination was taken from an overnight growth on MacConkey agar and suspended in $20 \mathrm{ml}$ of DM broth containing $0.63 \mathrm{~mm} \mathrm{~L}$-cysteine $\mathrm{HCl}$. After overnight incubation, the broth was centrifuged, the deposit was washed three times in saline and resuspended in saline before inoculation into $20 \mathrm{ml}$ of unsupplemented DM medium and then the above penicillin selection process was applied. The process was repeated with the unused portion of the deposit. The presence of cys ${ }^{-}$strains was determined according to colonial morphology, subculture and auxanography as described above. The organisms used in these experiments were: a prototrophic $E$. coli isolated from a stool sample from which a cys ${ }^{-} E$. coli was also isolated; $E$. coli NCTC 10418 as a prototrophic control; and partial and complete revertants to prototrophy (described above) of cys ${ }^{-} E$. coli $\mathrm{ECC}-1$ and ECC-13.

\section{Source of patient data and cys ${ }^{-}$strains}

A prospective study over 12 months was undertaken to confirm the incidence of cys ${ }^{-}$UTI and to assess certain features of the patients from whom these strains were isolated. Significant growths of Enterobacteriaceae from patients with UTI were recorded and such patients were placed into two groups, $<$ and $\geqslant$ 50 years of age. Plasma creatinine determinations (performed as part of standard patient investigations by means of an Olympus AU 500 autoanalyser, Tokyo, Japan) were recorded for these and other unselected patients with UTI due to cys ${ }^{-}$strains. A control group comprised patients aged $\geqslant 60$ years admitted for elective surgery but without evidence of UTI, uropathy or other serious illness. The reference range for the 
creatinine determinations was $0 \cdot 06-0 \cdot 11 \mathrm{mmol} / 1$. Tests for significance were performed with the $\chi^{2}$ test and Yate's correction for continuity.

\section{Source and preparation of chemicals}

Sodium sulphate was obtained from Merck and $\mathrm{CSH}$, used as a sulphite analogue, ${ }^{17}$ and all other chemicals were from Sigma. Cysteine was prepared from L-cysteine $\mathrm{HCl}$ immediately before use. ${ }^{18}$

\section{Results}

\section{Isolation of cys ${ }^{-}$strains from faecal samples}

Four cys ${ }^{-}$strains (three $E$. coli, one Klebsiella $\mathrm{sp}$.) were isolated from a faeces sample from each of four patients whose blood or urine, or both, was also infected with cys ${ }^{-}$isolates, but who had not received antibiotic therapy. In each case, the faecal and blood or urine strains were phenotypically identical. Cys

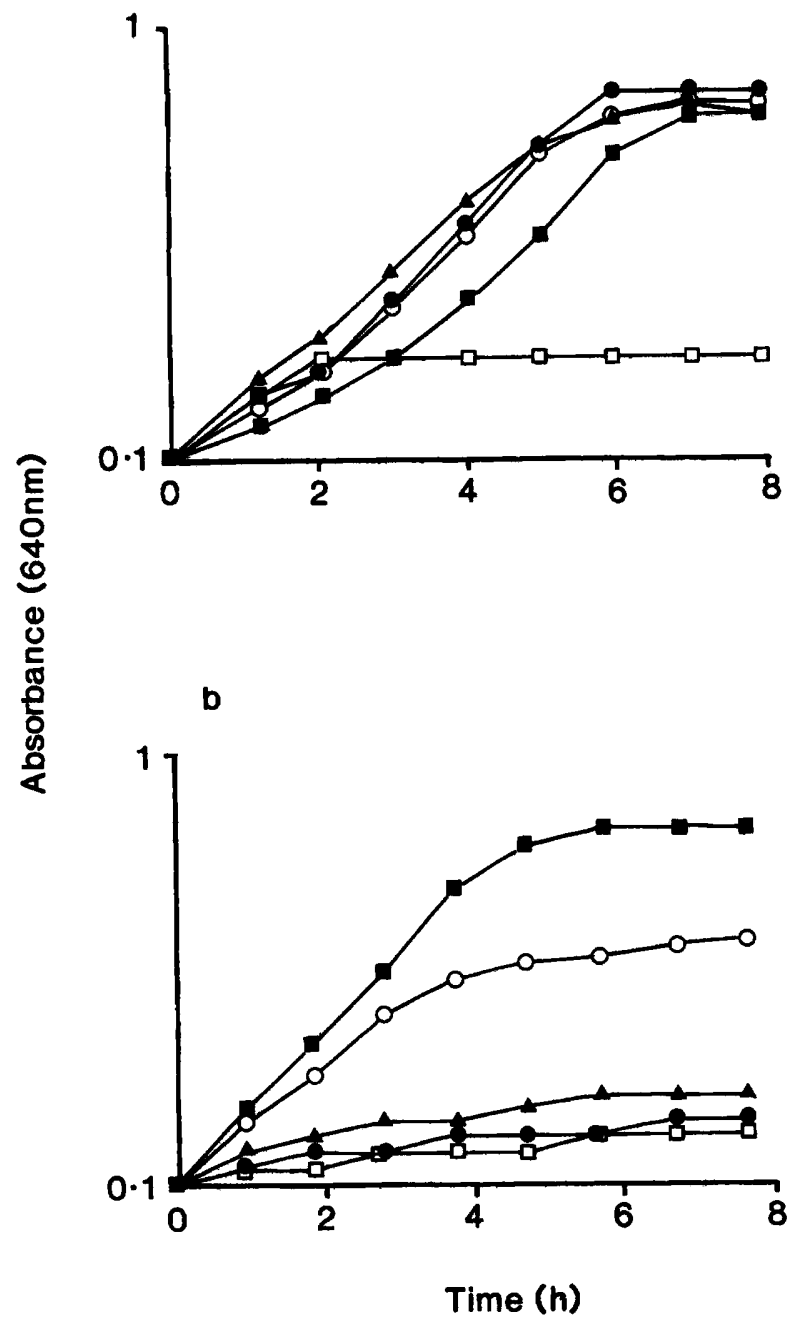

Fig. 1. Growth response of (a) E. coli NCTC 10418 and (b) a dwarf variant of E. coli ECC-1 in Pasternak's sulphur-free medium andthe same medium supplemented with intermediates of the sulphate assimilatory pathway: $-\square-$, blank; $-\mathbf{-}-$, sulphate; $-\mathbf{\Delta -}$, CSH; - O-, sulphide; - - -, cysteine. strains were not isolated from the stools of another seven patients also with cys ${ }^{-}$UTI. However, these patients had received an antibiotic, to which their cys ${ }^{-}$ isolate was sensitive, before collection of the stool sample. Only one cys ${ }^{-} E$. coli strain was isolated from the 100 stool samples from patients without evidence of UTI. It was from a 75-year-old female patient readmitted for an unrelated illness after recent sigmoid colectomy.

The five cys ${ }^{-}$strains were detected by both the direct and selective techniques. Although a significant reduction in prototrophic growth was obtained with the selective technique, and auxotrophs were much easier to distinguish, additional cys ${ }^{-}$strains were not detected by the use of this technique.

\section{Growth response of cys ${ }^{-}$strains to intermediates of the sulphate assimilatory pathway}

The growth responses in liquid medium of the phenotypic variants of $E$. coli cys $^{-}$ECC-1 and of a prototrophic control are shown in figs. 1 and 2. All

\section{a}

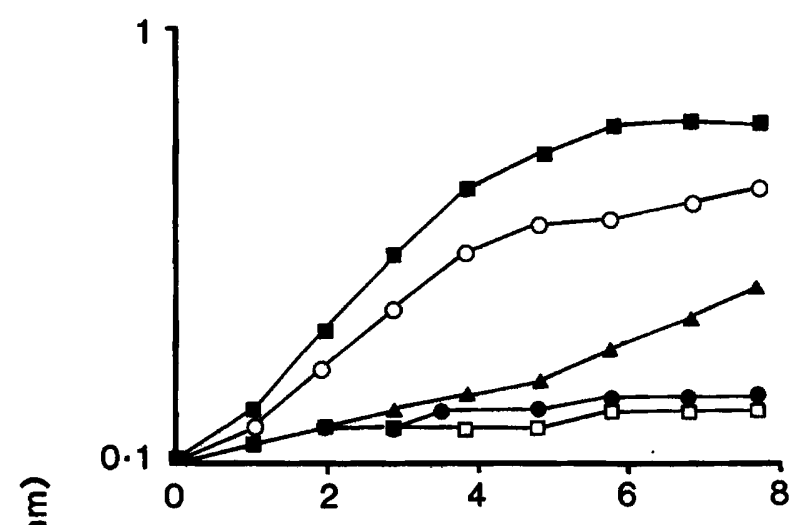

b

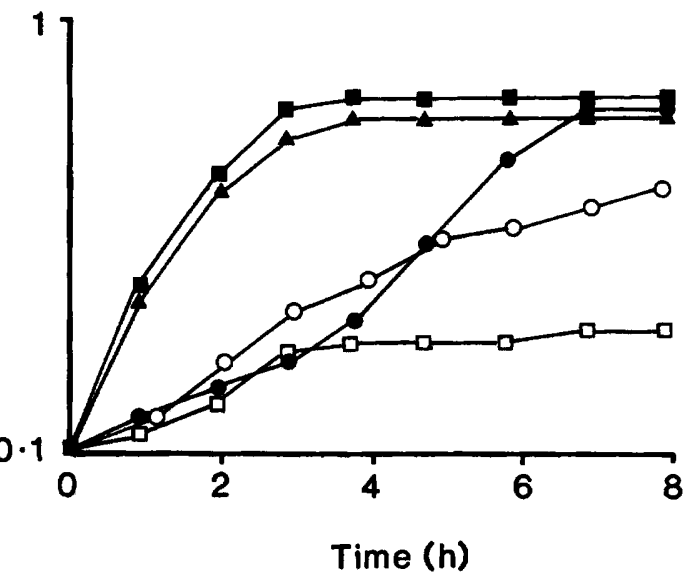

Fig. 2. Growth response of (a) partial and (b) complete revertants of $E$. coli ECC-1 in Pasternak's sulphur-free medium and the same medium supplemented with intermediates of the sulphate assimilatory pathway: $-\square-$, blank; $-\mathbf{-}$, sulphate; $-\mathbf{\Lambda}-$, $\mathrm{CSH} ;-\mathrm{O}-$, sulphide; - - - cysteine. 
cys $^{-}$variants were able to use cysteine and sodium sulphide. The dwarf and partial revertants were unable to use sulphate as a sulphur source, whereas the growth response to sulphate of the complete revertant closely resembled that of the prototrophic control. It can be seen also that the partial revertant differed from the auxotrophic "dwarf" variant in an increased capacity to use the sulphite analogue $\mathrm{CSH}$ as a sulphur source, but this was less than that of the complete revertant or the prototrophic control strain. It would seem that the partial revertants represent an intermediate stage between auxotrophy and prototrophy in which the metabolic defect demonstrated in the dwarf variant has been partially corrected. The results obtained with $E$. coli ECC-13 and $K$. pneumoniae KC6 were similar.

All 34 cys $A C D H$ strains grew on PM with $0.2 \mathrm{~mm}$ thiosulphate, but not on sulphur-free PM or on PM containing $\mathrm{Na}_{2} \mathrm{SO}_{4}$. Prototrophic control strains grew on all media except sulphur-free PM.

\section{Rate of partial and complete reversion to prototrophy of cys strains}

The reversion rates of four cys ${ }^{-} E$. coli and one cys Klebsiella strains to partial and complete prototrophy are shown in table I. The Klebsiella strains showed the highest reversion rate. The auxotrophic $E$. coli strain formed partial revertant colonies at a rate of $10^{-7}-10^{-8}$ and the rate of complete reversion was 100 -fold lower for two strains, but was not precisely determined for the remaining two isolates.

\section{Derivation of cys $^{-}$strains from prototrophic strains}

Dwarf colonies were obtained from partial and complete revertants grown in a cysteine broth. The cysteine requirement of these dwarf variants was confirmed auxanographically. Their detection was facilitated by the penicillin selection process, with cys ${ }^{-}$ dwarf colonies appearing after one cycle when partial revertants were used. However, distinction between cys $^{-}$auxotrophs and complete revertants was achieved only after repeated culture in cysteine broth followed by penicillin selection in cysteine-free medium. The cys $^{-}$strains derived in this way were able to revert to

Table I. Reversion rates to partial and complete prototrophy of four cys ${ }^{-} E$. coli and one cys- $K$. pneumoniae strains

\begin{tabular}{lcc}
\hline & \multicolumn{2}{c}{ Rate of reversion (cells $/ \mathrm{ml}$ ) } \\
\cline { 2 - 3 } Cysteine auxotroph & Partial & Complete \\
\hline E. coli ECC-1 & $2.2 \times 10^{-7}$ & $3.9 \times 10^{-9}$ \\
E. coli ECC-5 & $3.1 \times 10^{-8}$ & $>10^{-10}$ \\
E. coli ECC-13 & $4.6 \times 10^{-8}$ & $>10^{-11}$ \\
E. coli ECC-21 & $1.5 \times 10^{-8}$ & $1.4 \times 10^{-10}$ \\
K. pneumoniae KC-6 & $1.8 \times 10^{-6}$ & $4.4 \times 10^{-8}$ \\
\hline
\end{tabular}

Table II. Comparison between the plasma creatinine concentration in patients with UTI due to cys ${ }^{-} E$. coli and a control group aged $>60$ years

\begin{tabular}{cccc}
\hline & \multicolumn{2}{c}{ Number of patients } & $\begin{array}{c}\text { Number with raised } \\
\text { Age (years) }\end{array}$ \\
\cline { 2 - 3 } & infected & not infected & \\
\hline$>50$ & 27 & $\ldots$ & $15(55 \cdot 5)$ \\
$<50$ & 10 & $\ldots$ & $3(30)$ \\
$>60$ & $\ldots$ & 50 & $10(20)$ \\
\hline
\end{tabular}

prototrophy when cultured again on cysteine-deficient MacConkey medium. Cys ${ }^{-}$strains could not be derived from $E$. coli NCTC 10418. However, prototrophic wild-type $E$. coli, from the stool of a patient from whom a cys ${ }^{-} E$. coli was cultured simultaneously, yielded cys ${ }^{-}$auxotrophs after two cycles of growth and selection. This experiment was repeated on three separate occasions and each time a cys ${ }^{-}$strain was obtained. Again, the cys ${ }^{-}$strain reverted to prototrophy after subculture on cysteine-deficient media.

\section{Incidence of UTI with cys ${ }^{-}$strains}

A total of 1637 enterobacterial strains was isolated from patients with UTI in a 12-month period. Of these, $1106(67.6 \%)$ were E. coli, $226(13.8 \%)$ Klebsiella spp., 189 (11.5\%) Proteus-ProvidenciaMorganella spp. and the remaining $116(7 \cdot 1 \%)$ Enterobacter, Citrobacter or Serratia spp. Of the E. coli strains, $18(1.6 \%)$ were cys ${ }^{-}$, but only one $(0.4 \%)$ of the Klebsiella strains was cys ${ }^{-}$. Most (15 of 18) of the cys $^{-} E$. coli strains were isolated from patients aged $>50$ years and represented $2.7 \%$ of the $551 \mathrm{E}$. coli cultured from this age group. In patients $<50$ years of age, three $(0.5 \%)$ of 555 of $E$. coli were cys ${ }^{-1}$.

\section{Plasma creatinine concentrations in infected and uninfected patients}

Plasma creatinine concentrations were available for 37 patients with UTI due to cys ${ }^{-}$strains. They were compared in patients $<$ and $\geqslant 50$ years of age and in a control group of uninfected patients aged $>60$ years (table II). The proportion of patients in the group infected with cys ${ }^{-}$strains and with a raised plasma creatinine level (18 of 37) was significantly higher than in the control group $(0.001<\mathrm{p}<0.01)$ and this difference was greater in the infected subgroup $\geqslant 50$ years of age.

\section{Discussion}

This study confirmed that cys ${ }^{-}$E.coli strains represent a small percentage of all $E$. coli strains associated with UTI but that this is much higher in elderly patients. Although cys ${ }^{-}$Klebsiella strains were less 
common than the $1.5 \%$ previously reported, ${ }^{2}$ the smaller sample examined on this occasion may be responsible for the apparent discrepancy. It was also shown that, in common with other urinary pathogens, cys $^{-}$strains involved in UTI are derived from the bowel flora.

The effect of exogenous cysteine on the metabolic status of cys- strains was studied. In the absence of cysteine, cys $^{-}$strains reverted to prototrophy but remained or became auxotrophic in its presence. This was shown with cys ${ }^{-}$strains, their colonial variants in different stages of reversion to prototrophy, and with a prototrophic wild-type $E$. coli isolated from the stool of a patient in whom a cys ${ }^{-}$strain was also present.

$\mathrm{Cys}^{-}$strains examined in these and earlier studies were of the class cys $A C D H$ of Jones-Mortimer. ${ }^{13}$ This includes mutants deficient in the sulphate-utilising enzymes sulphate permease ( $c y s A$ ), adenosine $5^{\prime}$ phosphosulphate kinase (cys C), ATP sulphurylase (cys D) and 3'-phosphoadenosine 5'-phosphosulphate sulphotransferase (cys $H$ ). In this study, the capacity of cys $A C D H$ strains to utilise $0.2 \mathrm{~mm}$ thiosulphate was also demonstrated. This compound is metabolised at the level of sulphite by mutants deficient in the enzymes for sulphate reduction and activation..$^{16}$ However, as the cys $A$ gene controls the uptake of both sulphate and thiosulphate, ${ }^{19}$ it may be inferred from the growth response of these strains to thiosulphate that the permease system is operable and that the metabolic anomaly in these auxotrophs is related to the loss of functions controlled by cys $C$, cys $D$ or cys $H$ rather than cys $A$.

The genes cys $C$, cys $D$ and cys $H$ are located at $59 \mathrm{~min}$ on the $E$. coli chromosome and share the same location as cys $I$ and cys $J$ that code for the apohaemoprotein and apoflavoprotein components of sulphite reductase, respectively. ${ }^{20}$ The genes $c y s H$, cys $I$ and cys $J$ form an operon (cys $H I J$ ) which is separated from cys $C$ and cys $D$ by a "silent region" of c. $25 \mathrm{~kb}^{16}$ The findings described here in relation to the effects of cysteine on these clinically derived cys variants and the manner in which they utilise the sulphite analogue $\mathrm{CSH}$, are consistent with the involvement of an operon model in the regulation of cysteine synthesis described for laboratory-derived mutants. ${ }^{16,21}$ The improved growth response of the partial revertants in the presence of CSH may be explained by the gradual derepression of the cys $H I J$ operon in the absence of the product of the sulphate assimilatory pathway. Cysteine can be postulated as playing the role of repressor in this model. As a consequence, there is an energy saving for the cell, since the assimilatory pathway requires two mols of ATP to activate sulphate.

The postulated operon mechanism in cys $^{-}$strains provides the basis of a possible explanation for their selection in vivo, if levels of cysteine over and above those present from dietary sources occur in the bowel. This possibility led to a consideration of some patient parameters to ascertain whether this is indeed the case. Patients with impaired renal function retain sulphurcontaining as well as nitrogen-containing compounds, and the plasma concentrations of taurine, cystine and cysteine-homocysteine are consistently raised in patients with a raised plasma creatinine.$^{8-10} \mathrm{~A}$ preliminary examination of data from patients infected with cys $^{-}$strains confirmed that they comprise a mostly older age group and that there is an association between the isolation of cys ${ }^{-}$strains and reduced renal function. However, considerable difficulties are associated with the proper assay of cystine and cysteine in plasma $^{22}$ and these, together with the contentious nature of the methodology involved, ${ }^{23,24}$ have prevented confirmation of this inferred accumulation of cysteine-related compounds in the plasma or bowel lumen of these patients. Furthermore, there is no information about the relationship between plasma and bowel concentrations of these compounds, but at least some of these substances are excreted in the bile and their concentration in the plasma and bowel lumen are known to equilibrate to some extent. However, if raised concentrations of sulphur-containing compounds, that can be assimilated by cys strains, actually appear in the bowel through this or other mechanisms, the selection of cys ${ }^{-}$strains may also take place in the bowel. This would be in the type of patient in whom these infections predominantly occur and it should be recalled that the bowel is the source of most uropathogens. This hypothesis awaits further clarification.

\section{References}

1. Borderon E, Horodniceanu T. Metabolically deficient dwarfcolony mutants of Escherichia coli: deficiency and resistance to antibiotics of strains isolated from urine culture J Clin Microbiol 1978; 8: 629-634.

2. McIver CJ, Tapsall JW. Characteristics of cysteine-requiring strains of Klebsiella isolated from urinary tract infections. J Med Microbiol 1988; 26: 211-215.

3. Tapsall JW, McIver CJ. Septicaemia caused by cysteinerequiring isolates of Escherichia coli. J Med Microbiol $1986 ; 22$ : 379-382.

4. McIver CJ, Tapsall JW. Cysteine requirements of naturally occurring cysteine auxotrophs of Escherichia coli. Pathology $1987 ; 19$ : 361-363

5. McIver CJ, Tapsall JW. Assessment of conventional and commercial methods for identification of clinical isolates of cysteine-requiring strains of Escherichia coli and Klebsiella species J Clin Microbiol 1990; 28: 1947-1951.

6. McIver CJ, Tapsall JW. In vitro susceptibilities of clinical isolates of cysteine-requiring Escherichia coli to 12 antimicrobial agents. Antimicrob Agents Chemother 1991; 35 995-997.

7. Gillespie WA. Biochemical mutants of coliform bacilli in infections of the urinary tract. $J$ Pathol Bacteriol 1952; 64 : 551-557.

8. Wilcken DEL, Gupta VJ. Sulphur containing amino acids in chronic renal failure with particular reference to homocysteine and cysteine-homocysteine mixed disulphide. Eur J Clin Invest 1979; 9: 301-307. 
9. Wilcken DEL, Gupta VJ, Betts AK. Homocysteine in the plasma of renal transplant recipients: effects of co-factors for methionine metabolism. Clin Sci 1981; 61 : 743-749.

10. Wilcken DEL, Gupta VJ, Reddy SG. Accumulation of sulphurcontaining amino acids including cysteine-homocysteine in patients on maintenance haemodialysis. Clin Sci 1980; 58: $427-430$.

11. Davis BD. Isolation of biochemically deficient mutants of bacteria by penicillin. J Am Chem Soc 1948; 70: 4267

12. Davis BD, Mingioli ES. Mutants of Escherichia coli requiring methionine or vitamin B12. J Bacteriol 1950; 60: 17-28.

13. Jones-Mortimer MC. Positive control of sulphate reduction in Escherichia coli. Isolation, characterization and mapping of cysteineless mutants of $E$. coli K12. Biochem $J 1968$ 110: $589-595$.

14. Pasternak CA. Sulphate activation and its control in Escherichia coli and Bacillus subtilis. Biochem J 1962; 85: 44-49.

15. Mizobuchi, K., Demerec M, Gillespie DH. Cysteine mutants of Salmonella typhimurium. Genetics 1962; 47: 1617-1627.

16. Kredich NM. Biosynthesis of cysteine. In: Neidhardt FC (ed) Escherichia coli and Salmonella typhimurium: cellular and molecular biology, vol 1. Washington, DC, American Society for Microbiology. 1987: 419-428.
17. Dreyfuss J, Monty KJ. The biochemical characterization of cysteine-requiring mutants of Salmonella typhimurium. $J$ Biol Chem 1963; 238: 1019-1024.

18. Postgate JR. The examination of sulphur auxotrophs: a warning. J Gen Microbiol 1963; 30: 481-484.

19. Parra F, Britton CP, Castle C, Jones-Mortimer MC, Kornberg HL. Two separate genes involved in sulphate transport in Escherichia coli K12. J Gen Microbiol 1983; 129: 357-358.

20. Bachmann BJ. Linkage map of Escherichia coli $\mathrm{K} 12$, edition 8. Microbiol Rev 1990; 54: 130-197.

21. Jacob F, Monod J. Genetic regulatory mechanisms in the synthesis of proteins. J Mol Biol $1961 ; 3$ : 318-356.

22. Perry TL, Hansen S. Technical pitfalls leading to errors in the quantitation of plasma amino acids. Clin Chim Acta 1969; 25: 53-58.

23. Cooper JDH, Turnell DC, Green B, Wright DJ, Coombes EJ Why the assay of serum cystine by protein precipitation should be abandoned. Ann Clin Biochem 1988; 25: 577582.

24. Schneider JA, Dalton $R N$. Why the assay of serum cystine by protein precipitation and chromatography should be abandoned. Ann Clin Chem 1989; 26: 305-306. 